\title{
ECONOMIC GROWTH BY MEANS OF INTERSPECIFIC FUNCTIONAL RESPONSE OF CAPITAL-LABOUR IN DYNAMICAL SYSTEM
}

\author{
OYOON ABDUL RAZZAQ ${ }^{1}$, NAJEEB ALAM KHAN2, , NOOR UL AIN2 , MUHAMMAD \\ AYAZ 2 \\ ${ }^{1}$ Department of Humanities \& Social Sciences, Bahria University, Karachi 75260, Pakistan \\ 2Department of Mathematics, University of Karachi, Karachi 75270, Pakistan
}

*Corresponding author: njbalam@yahoo.com

ABSTRACT. Physical capital and labour force are two major factors of any economy, which play a key role in its growth. The association of these two components with each other is also a matter of study, which is carried out in this endeavour by means of an ecological system with Holling-type II function. The governing model is an avantgrade approach for economic theory as its equilibrium states and the stability analysis so obtained, referrer to different economic states with detail information about the capital-labour interaction. This novel assessment also contributes a significant way to scrutinize the capability of labours on consuming time on the capital and the efficiency of capital on processing output. Moreover, different patterns and cyclic behaviour of the Cobb-Douglas and constant-elasticity production functions, for different steady and oscillating states of the system are also provided comparatively. In addition, a numerical example is also discussed graphically with economic significance. These measurements will consequently keep the production cycle moving and so sustain the economic growth.

Received 2019-04-24; accepted 2019-05-22; published 2019-07-01.

2010 Mathematics Subject Classification. 91B02.

Key words and phrases. production function; Holling-type II function; equilibrium points; economic growth.

(C)2019 Authors retain the copyrights of their papers, and all open access articles are distributed under the terms of the Creative Commons Attribution License. 


\section{Introduction}

There is a large number of literature that has revealed different exogenous and endogenous causes and other active censures, which might destroy or stable the well-ordered economy of a country. The neo-classical theory of economic growth was firstly developed by Robert Solow as an alternative to the Harrod-Domar model of growth. Along with the labour and capital, he also added that the growth of any country comes from adding more ideas and new technologies. Recently, many types of research are found that illustrated these factors in production functions [1-9]. Fundamentally, these models accumulate many major assumptions of capital and labour in the production function, and as a consequence in the long-term economic growth. For instance,

- Sustain rise in capital investment increases the ratio of capital to labour, which temporarily increases the growth rate.

- Increase in the labour supply in addition to a higher level of productivity of labour and capital raises the trend of growth rate.

- The declination of the marginal product of additional units of capital causes a long-term growth path with the same growth rate of GDP of an economy.

- When the growth rate of output, capital and labour force is the same and so output per worker and capital per worker are constant, the steady-state growth path is reached.

- Different pace of technological changes between countries will also show much variation in the growth rates.

The present endeavour shows a ground-breaking approach to study the upshots of a production function that stem from the interaction of the capital and the labour force with the help of the ecological assumptions. We structured the dynamical system of predator-prey as the labour force (the output from the workers) and physical capital (machines), respectively. The predator-prey relationship is deep-rooted in nature and now it has become one of the inveterate concept to study the interactions among species and their environment. After the contrivance of theoretical ecology by Lotka [10] and Volterra [11], the models, named as Lotka-Volterra equations, played an important role in different fields of applied sciences [12-15]. The novelty in this attempt is the inclusion of Holling-type II function [16-20] in the system, which can effectively measure the functional response between the capital and the labour force. The existence of competitive interaction between these two factors produces a major change in the 
production process that yields a salient change in economic growth. Using these moulds, the economic cycle that possibly occurs as a result of the interaction is also sketched in Fig. 1. Each equilibrium point of the dynamical system is discussed with its stability and economic significance. Different plots of some production functions [21-24] are also added to study the theory pictorially. The inventive incorporation of ecological concept in the economic growth theory will definitely make the mainstream economist think diversely while making economic measurements.

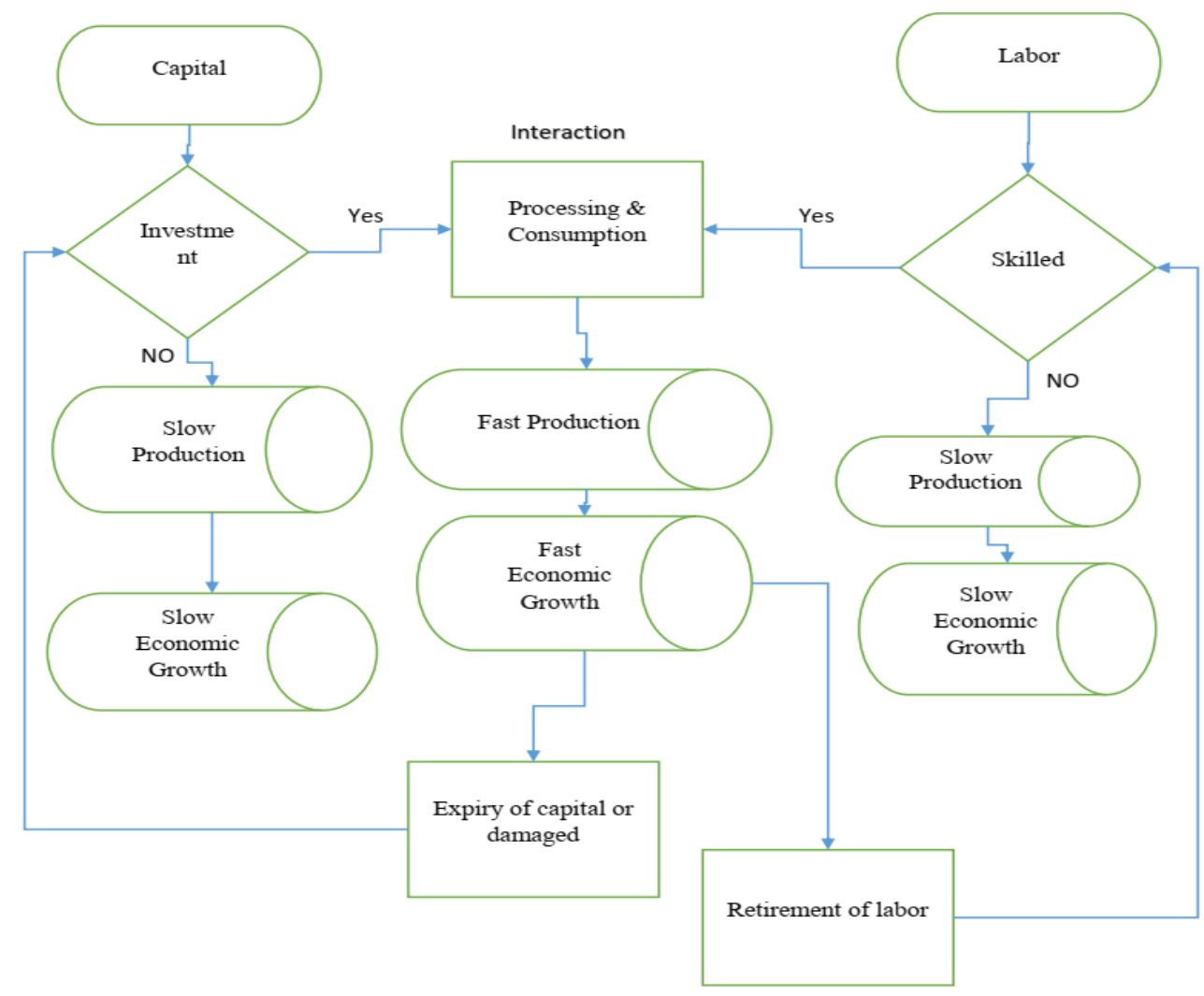

Figure 1: Pictorial view of different circumstances with capital-labour interaction

\section{Mathematical Modelling and Equilibria}

Nowadays, economic growth is widely studied to estimate the social stability of a country around the globe. Most of the models, which are used for this purpose, are based on the production function that comprehends the exponential product of physical capital and labour force. The term physical capital includes things like machines, computers and other equipment that are used in the production process. Whereas the labour force is meant to be the performance of skilled and unskilled activities of human workers. The previous models lack 
many repercussions, which occur during the output production. For instance, the effects of death, retirement or termination etc. of labours in a particular time period. Similarly, the time consumed operating a machine, its expiry etc. are the key factors that actually affect the performance of producing output in a targeted duration. These upshots affect the production progress on the individual level, which gradually leads to fluctuations in GDP of a country. Innovatively, considering all these facts, we take advantage of the well-known ecological systems, which sorts out dynamics of such ups and downs. The novelty in the model is that the labours are assumed as predators, which use the physical capitals as their prey in combination with Holling type II function.

\subsection{Model Manifestation}

Let, $K(t)$ and $L(t)$ represent the physical capital and labour force. Then the dynamics of these two species can be studied through the following system of equations, in conjunction with the Holling type II function [3-9, 16-20] as,

$$
\begin{aligned}
& \frac{d K(t)}{d t}=s f(K(t))-\beta \Psi_{I I}(t)-\delta K(t), \\
& \frac{d L(t)}{d t}=r g(L(t))+\gamma \Psi_{I I}(t)-\sigma L(t),
\end{aligned}
$$

With the initial conditions as,

$$
K(0)=K_{0} \text { and, } L(0)=L_{0}
$$

In system (1), $s$ and $r$ show the capital investment and population of labours, respectively.

Here, we assume $f(K(t))=K(t)\left(1-\frac{K(t)}{\eta_{1}}\right)$ and $g(L(t))=L(t)\left(1-\frac{L(t)}{\eta_{2}}\right)$, as the logistic growth of capital and labours, where $\eta_{1}$ and $\eta_{2}$ define the maximum populations of capital and labours sustained by the environment. Whereas, $\beta$ andextent to which the are the $\gamma$ availability of the labour force affects the growth of capital and vice versa, accordingly. The Holling type II function, $\Psi_{I I}(t)$, is outlined as,

$$
\Psi_{I I}(t)=\frac{\alpha K(t) L(t)}{1+\alpha H K(t)}
$$

whereor the at which the labour come into contact with equipment the rate examines $\alpha$ learning time and $H$ measures the handling time or the average time spent to process a capital 
for producing output. Finally,and tal expiry of the capi or explain the depreciation $\sigma$ nd a $\delta$ the retirement, dismissal or death of labour, respectively.

This advanced concept in the above system can be easily understood by considering different states of economic growth of any country. For instance, let the investment on machinery is increased while keeping the appointments of new labours constant. The existing labours are then allocated on appropriate machines for producing output. Each labour has to operate multiple machines at a time. This is because the proportion of machines per labour decreases as capital density increases. Additionally, at a very high level of investment on machines, labours require very little time waiting or searching for a machine and spend almost all their time in processing and producing output. Effectiveness of labour is then satiated and the number of invested capital reaches a plateau. This will in return brings out a positive effect on the growth rate of the labour force as it increases the labour efficiency to produce output more effectively.

\subsection{Equilibria and Stability Illustration}

Equilibria are the constant solutions to the system and are attained by taking the derivatives of the functions equal to zero, coupled with the stability analysis which is investigated through the eigenvalues of the Jacobian matrix at a particular equilibrium point. Generally, if the matrix has a positive real part or multiple zeroes then the equilibrium point is unstable for both cases. Negative real parts of all eigenvalues conclude the point to be locally asymptotically stable. Moreover, if the Jacobian matrix has negative real eigenvalues along with a pair of purely imaginary eigenvalues, then a Hopf bifurcation point might occur. The linearized stability test fails if it has negative real eigenvalues with exactly one zero eigenvalues.

Now, consider Eq. (1) with $\frac{d K(t)}{d t}=0$, and let the Jacobian matrix for an equilibrium $\frac{d L(t)}{d t}=0$ point $\left(K^{*}, L^{*}\right)$ be,

$$
\mathfrak{I}\left(K^{*}, L^{*}\right)=\left[\begin{array}{cc}
s\left(1-\frac{2 K^{*}}{\eta_{1}}\right)-\frac{\alpha \beta L^{*}}{\left(1+\alpha H K^{*}\right)^{2}}-\delta & -\frac{\alpha \beta K^{*}}{1+\alpha H K^{*}} \\
\frac{\alpha \gamma L^{*}}{\left(1+\alpha H K^{*}\right)^{2}} & r\left(1-\frac{2 L^{*}}{\eta_{2}}\right)+\frac{\alpha \gamma K^{*}}{1+\alpha H K^{*}}-\sigma
\end{array}\right],
$$

Then the governing system (1) has the following equilibrium points arising in different circumstances. 


\subsubsection{Trivial Equilibrium Point}

On substituting the trivial equilibrium point $e_{0}(0,0)$ in the Jacobian matrix and simplifying, we get the eigen values $(s-\delta, r-\sigma)$, which shows the $e_{0}$ to be locally asymptotically stable if, $0<s<\delta$ and. $0<r<\sigma$

\subsubsection{Predator-free Equilibrium Point}

The predator-free equilibrium point is attained as $e_{1}\left(\frac{\eta_{1}(s-\delta)}{s}, 0\right)$. On substituting $e_{1}$ in the Jacobian matrix and simplifying, we get the eigen values $\left(\delta-s, r-\sigma-\frac{\alpha \gamma \eta_{1}(\delta-s)}{s+H s \alpha \eta_{1}-H \delta \alpha \eta_{1}}\right)$, which shows the $e_{1}$ to be locally asymptotically stable if, $s>\delta$ and. $r \leq \frac{-\alpha \eta_{1} \gamma+\sigma+H \alpha \eta_{1} \sigma}{1+H \alpha \eta_{1}}$

\subsubsection{Prey-free Equilibrium Point}

The calculated prey free equilibrium point is $e_{2}\left(0, \frac{\eta_{2}(r-\sigma)}{r}\right)$. For this point, the Jacobian matrix produces the eigenvalues $\left(\sigma-r, s-\delta+\frac{\alpha \beta \eta_{2}(\sigma-r)}{r}\right)$, which shows the $e_{2}$ to be locally asymptotically stable if $0<s<\frac{r \delta+\alpha \beta \eta_{2}(r-\sigma)}{r}$ and. $r>\sigma$

\subsubsection{Coexistence Equilibrium Point}

Let the coexistence equilibrium point beuch that, $\mathrm{s}_{3}(\bar{K}, \bar{L})$

$$
\begin{aligned}
\bar{K}= & \frac{1}{F}\left(-G+\left(\frac{2}{A+\sqrt{A^{2}-4\left(G^{2}+F M\right)^{3}}}\right)^{1 / 3}\left(G^{2}+F M\right)+\left(\frac{A+\sqrt{A^{2}-4\left(G^{2}+F M\right)^{3}}}{2}\right)^{1 / 3}\right), \\
\bar{L}= & \frac{1}{\alpha \beta}(s-\delta)-\frac{G}{\alpha \beta \eta_{1}} \frac{\left(s+H s \alpha+H s \alpha \eta_{1}+H \alpha \delta \eta_{1}\right)}{F} \\
& +\frac{1}{\alpha \beta \eta_{1}} \frac{\left(s+H s \alpha+H s \alpha \eta_{1}+H \alpha \delta \eta_{1}\right)}{F}\left(\frac{A+\sqrt{A^{2}-4\left(G^{2}+F M\right)^{3}}}{2}\right)^{1 / 3}\left(G^{2}+F M\right) \\
& +\frac{1}{\alpha \beta \eta_{1}} \frac{\left(s+H s \alpha+H s \alpha \eta_{1}+H \alpha \delta \eta_{1}\right)}{F}\left(\frac{A+\sqrt{A^{2}-4\left(G^{2}+F M\right)^{3}}}{2}\right)^{1 / 3},
\end{aligned}
$$


where,

$$
\begin{aligned}
& A=-2 H^{3} r^{3} \alpha^{3}\left(s+H s \alpha \eta_{1}-H \delta \alpha \eta_{1}\right) H^{2} \delta^{2} \alpha^{2} \eta_{1}^{2} \\
& -2 H^{3} r^{3} \alpha^{3}\left(s+H s \alpha \eta_{1}-H \delta \alpha \eta_{1}\right)\left(s+H s \alpha \eta_{1}\right)^{2} \\
& +H^{4} r^{3} \alpha^{4} s \eta_{1}\left(s+H s \alpha \eta_{1}-H \delta \alpha \eta_{1}\right)\left(4 \delta\left(1+H \alpha \eta_{1}\right)+9 \alpha \beta \eta_{2}\left(s+H s \alpha \eta_{1}-H \delta \alpha \eta_{1}\right)\right) \\
& -9 H^{3} r^{2} \alpha^{5} s \beta \eta_{1} \eta_{2}\left(H \delta \alpha \eta_{1}(\gamma-H \sigma)+s\left(\gamma\left(2-H \alpha \eta_{1}\right)+H\left(1+H \alpha \eta_{1}\right) \sigma\right)\right) \text {, } \\
& F=3 H^{2} r s \alpha^{2}, G=-2 H r s \alpha+H^{2} r s \alpha^{2} \eta_{1}-H^{2} r \alpha^{2} \delta \eta_{1} \\
& M=-r s+2 H r s \alpha \eta_{1}-2 H r \alpha \delta \eta_{1}-H r \alpha^{2} \beta \eta_{1} \eta_{2}-\alpha^{2} \beta \gamma \eta_{1} \eta_{2}+H \alpha^{2} \beta \eta_{1} \eta_{2} \sigma,
\end{aligned}
$$

The $e_{3}$ will exists, if $A^{2}>4\left(G^{2}+F M\right)$ or $\left(G^{2}+F M\right)<0$. On substituting $e_{3}$ in Eq. (3), we get the following characteristic equation,

$$
\lambda^{2}+D_{1} \lambda+D_{2}=0
$$

with $\quad D_{1}=-r-s+\delta+\sigma+\bar{L}\left(\frac{2 r}{\eta_{2}}+\frac{\alpha \beta}{1+H \alpha \bar{K}}\right)-\bar{K}\left(-\frac{2 s}{\eta_{1}}+\frac{\alpha \gamma}{1+H \alpha \bar{K}}+\frac{H \alpha^{2} \beta \bar{L}}{(1+H \alpha \bar{K})^{2}}\right) \quad$ and

$$
D_{2}=\frac{\alpha^{2} \beta \gamma \bar{K} \bar{L}}{(1+H \alpha \bar{K})^{3}}+\left(r-\sigma+\frac{\alpha \gamma \bar{K}}{1+H \alpha \bar{K}}-\frac{2 r \bar{L}}{\eta_{2}}\right)\left(s-\delta-\frac{\alpha \beta \bar{L}}{1+H \alpha \bar{K}}+\bar{K}\left(-\frac{2 s}{\eta_{1}}+\frac{H \alpha^{2} \beta \bar{L}}{(1+H \alpha \bar{K})^{2}}\right)\right)
$$

which has the roots,

$$
\lambda=\frac{-D_{1} \pm \sqrt{D_{1}^{2}-4 D_{2}}}{2}
$$

Sotherwise, o $D_{1}^{2}-4 D_{2}>0$ and $i=1,2$ or $\mathrm{f} D_{i}>0$, is locally asymptotically stable if $e_{3}$ unstable with a complex conjugate pair and then a Hopf bifurcation will lead to a limit cycle.

\section{Numerical Discussion with Economic Significance}

In this section, we have discussed the aforementioned model for some numerical values of the parameters and attained different graphical demonstration using the Mathematica 11.0 software program. The Cobb-Douglas and constant-elasticity of production functions [21-24] are also portrayed to study the effects on the economic growth that takes place by means of the functional responses of physical capital and labour force. Mathematica 11.0 has a built-in program of the well-known fifth-fourth Runge Kutta method. Nowadays, this software has gained significant importance because of its friendly user interface and capability of solving any 
kind of mathematical model. The appendix contains the code of this program made for illustrating the governing model numerically and graphically.

A pictorial view of the system (1) is obtained by taking $s=2, r=2, \eta_{1}=3, \eta_{2}=5, \beta=1$ $\gamma=1, \alpha=2, \delta=4, \sigma=4$,in shown, as $L(0)=1$ and $K(0)=1$, with initial conditions $H=5$

Fig. 2. The existence of trivial equilibrium point can be seen clearly in this figure for a long-term. This is assumed as the production phase in the absence of technical machinery and skilled labours, as comprehensively discussed in the Cobb-Douglas model [21-22]. It also explains that if depreciation of capital and termination of labours become greater than the investment and appointment of new labours, accordingly, both factors will extinct after a particular time. As a result, the economic growth will either stop or reach the underperformance stage.

Fig. 3 apparently describes the prey free equilibrium stage for the values, $s=2, r=4, \eta_{1}=3$, $\eta_{2}=5, \gamma=6, \beta=1, \alpha=8, \delta=4, \sigma=2,$. The, with the same initial conditions $H=15$ condition evidently states that the increase in the number of employees requires more investment in the capital. Thus, if further investment is not made, the rate becomes inversely proportional to the increasing number of labours and the deficiency of physical capital occurs. Therefore, on continuous usage of the available capitals, after reaching a particular time, it will suffer devastation and no more machinery will exist. It is the plateau when a change in the economy is due to the effective endeavours of labours to produce output without using advanced types of machinery. Examples can be taken as the countries that are below the line of underdevelopment, which have a deficiency in the latest technical developments. Subsequently, the economic growth will either stop or move slowly as compared to the developed countries that accompany high technologies to boost their production growth.

The predator-free equilibrium is shown in Fig. 4 for the parameters taken as $s=5, r=002$, $\eta_{1}=3, \eta_{2}=5, \gamma=6, \beta=1, \alpha=8, \delta=4, \sigma=2$ " with the aforementioned initial $H=3$ conditions. It elucidates that there is an investment on new latest technologies but due to the lack of skilled and expert labours, this will automatically decrease the efficiency of labour force towards the production, which in return decreases the economic growth.

Fig. 5 portrays the oscillations that illustrate the unstable state of coexistence equilibrium for the parameters taken as $s=3, r=2, \eta_{1}=1, \eta_{2}=3, \gamma=6, \beta=1, \alpha=8, \delta=2, \sigma=3, H=3.5$ with the same initial conditions. It states the performance of both the factors with the rate of 
investment on capital greater than the rate of labour appointments. This will create fluctuations in the economic activity of a country.

Furthermore, Figs. 6-9 demonstrate the production for different stages of equilibria of the capital and labour force for the time $t \in[0,100], \eta_{1}=2, \eta_{2}=3, \gamma=6, \beta=1, \alpha=8,, H=3.5$ and different values of $s, r, \delta$,known -the wellplotting $\mathrm{s}$ are achieved by . These figure $\sigma$ Cobb-Douglas production function,

$$
P(K, L)=\Omega K(t)^{\rho} L(t)^{1-\rho},
$$

with factor productivity $\Omega=1$ and $\rho=0.4$. Here, the values of $K(t)$ and $L(t)$, generated through the dynamical system (1), are substituted in Eq. (4) to attain the curves. To the best of our knowledge, in most of the literatures [21-22] the parametric graph of Eq. (4) is usually plotted for constant rate of labour force. Evidently, those curves contradict the reality that are the actual cause of rise and fall of economic cycle. Here, comparative patterns of the Eq. (4) are illustrated for distinct configurations of capital and labour. Fig. 5 shows an increasing production process when investment and growth rate of labours are equal and so is the depreciation and retirement rate. In Fig. 6 a curvy rise and fall can be seen in the production function, which is attained by taking the investment level less than growth of the labour and depreciation of the capital greater than the retirements of the labours. On the other hand, production process is found increasing in Fig. 7 in a nonlinear manner for the case when the investment level is greater than the growth of the labour and also depreciation rate is taken greater than retirements. Furthermore, when investment is increased as compared to the growth of labors and depreciation rate is decreased against the retirements of the labors, a cyclic production progress is attained, as shown in Fig. 8.

In addition, comparison plots of different production functions proposed in the literature [2124] are also demonstrated in Figs. 10(a-c)-12(a-c). These graphs are also attained consequently through the solutions of the dynamical system (1) for different values of $\alpha$ and $H$, with $\eta_{1}=2$ ${ }^{,} \eta_{2}=3, \gamma=6, \beta=1, \delta=2, \sigma=3, r=2$, is production functionDouglas -The Cobb . $s=3$ shown in Figs. 10a, 11a and 12a, the constant-elasticity of production function [23],

,$P(K, L)=\Pi(1.0183)^{t}\left(\omega K(t)^{-\rho}+(1-\omega) L(t)^{-\rho}\right)^{-1 / \rho}$ 
is plotted in Figs. 10b, $11 \mathrm{~b}$ and $12 \mathrm{~b}$ for $\Pi=0.584, \omega=0.519$ and $\rho=0.756$. While Figs. 10c, $11 \mathrm{c}$ and $12 \mathrm{c}$ represent the production function $[23,24]$,

$$
P(K, L)=\Pi\left(\omega K(t)^{-\rho}+(1-\omega) L(t)^{-\rho}\right)^{-1 / \rho},
$$

for $\Pi=1, \omega=0.519$ and $\rho=0.756$, where $\Pi$ is the efficiency parameter, $\omega$ is functional distribution parameter and $\rho$ is the elasticity substitution parameter in Eqs. (5) and (6). The unstable open regions are clearly found in Figs. 10(a-c) of each production function, respectively, for $\alpha=8$ and $H=1$. Figs. 11a and 11c, illustrate small logarithmic spirals for the parameters $\alpha=2$ and $H=2$, whereas Fig. 11c starts with a constant line and after some time goes round. Furthermore, when $\alpha=8$ and $H=3.5$, Figs. 12a and 12c portrait a single rotation, while $12 \mathrm{~b}$ produces multiple rotations, with the contraction in the open unstable region.

\section{Conclusion}

In this paper, we studied the economic growth by means of the ecological system in conjunction with Holling-type II function. The discussions were successfully made by relating the biological terms of the system with the economical parameters and defining preys and predators as the capital and labour force, accordingly. All the possible equilibrium points of the ecological structure were analysed according to economic significance. Many production functions have been found in literature, which has been used to study economic growth. Here, we took some among them and studied the effect of functional response of physical capital and labour force on these production functions.

We figured out the following outcomes that are surely beneficial for the mainstream economists for different aspects of economic studies.

- Each of the constant solutions shows different states of the economy that might occur endogenously or exogenously.

- Generated production functions show different curves for each case of equilibrium points of physical capital and labour force.

- The prey-free steady-state interprets the economy that is less developed technologically. Here, exogenous causes might affect the rate of investment on the latest equipment, which can boost the efficiency of labour output.

- Predator-free state reflects the economy that lacks skilled labours, i.e. in the absence of competent labours, the increasing rate of investment on the latest types of machinery will not be adequately beneficial for economic growth. This state might also be due to 
the migration of educated and proficient labours to other countries for the sack of high wages etc. Thus to this end, proponents of endogenous causes might play a key role in this regard.

- The coexistence fluctuations are considered as the ups and downs in the growth of capital and efficiency of labours force. The production function of such economies yields cyclic behaviour. These cycles keep the economic ball rolling of any country.

- The functional response of physical capital and labour is evidently seen to be a major characteristic of the production function, as these factors together may create a positive effect on economic growth.

- The time consumed by the labours on each equipment can modify the output of the labour force factor, which overall enrich the production progress.

- The Holling-type II function helps to evaluate the handling time and searching efficiency of the labours for the physical capitals.

- Stability conditions at each state can balance the investment and depreciation on capital, similarly growth in excessive appointments and retirement or dismiss of professional labours.

In future, some more interspecific interactions between different economic components will be discussed in addition to the imprecise theory.

\section{Appendix}

solex $=\operatorname{NDSolve}[\{\mathrm{D}[\mathrm{K}[\mathrm{t}], \mathrm{t}]==\mathrm{s} \mathrm{K}[\mathrm{t}](1-\mathrm{K}[\mathrm{t}] / \backslash[$ Eta $] 1)-(\backslash[$ Beta $] \backslash[$ Alpha $] \mathrm{K}[\mathrm{t}] \mathrm{L}[\mathrm{t}]) /(1+$ $\backslash[$ Alpha] H K[t]) - \[Delta] K[t], D[L[t], t] == r L[t] $(1-\mathrm{L}[\mathrm{t}] / \backslash[$ Eta $] 2)+(\backslash[$ Gamma $] \backslash[$ Alpha] $\mathrm{K}[\mathrm{t}] \mathrm{L}[\mathrm{t}]) /(1+\backslash[$ Alpha] $\mathrm{H} \mathrm{K}[\mathrm{t}])-\backslash[$ Sigma $] \mathrm{L}[\mathrm{t}], \mathrm{K}[0]==1, \mathrm{~L}[0]==1\},\{\mathrm{K}[\mathrm{t}], \mathrm{L}[\mathrm{t}]\},\{\mathrm{t}, 0,100\}]$

Plot $[$ Evaluate $[\{\mathrm{K}[\mathrm{t}], \mathrm{L}[\mathrm{t}]\} \quad /$. solex $], \quad\{\mathrm{t}, 0,5\}$, PlotStyle $\rightarrow\{$ Blue, Dashed $\}$, Frame $\rightarrow$ True, PlotTheme $\rightarrow$ "Business", AxesStyle $\rightarrow$ Directive[Black, 12], FrameLabel $\rightarrow\{$ "Time(t)", "Population(K[t],L[t])"\}, PlotLegends $\rightarrow\{$ "K[t]", "L[t]"\}]

ParametricPlot3D[Evaluate $\left[\left\{(\mathrm{K}[\mathrm{t}])^{\wedge} 0.4 \quad(\mathrm{~L}[\mathrm{t}])^{\wedge} 0.6, \quad(\mathrm{~K}[\mathrm{t}]), \quad(\mathrm{L}[\mathrm{t}])\right\} \quad / . \quad\right.$ solex $], \quad\{\mathrm{t}, \quad 0, \quad 100\}$, PlotTheme $\rightarrow$ "Marketing", PlotRange $\rightarrow$ All, AxesLabel $\rightarrow\{$ "P[K,L]", "K[t]", "L[t]"\}] 
solH1 = ParametricNDSolve $[\{\mathrm{D}[\mathrm{K}[\mathrm{t}], \mathrm{t}]==\mathrm{s} \mathrm{K}[\mathrm{t}](1-\mathrm{K}[\mathrm{t}] / \backslash[\mathrm{Eta}] 1)-(\backslash[$ Beta $] \backslash[$ Alpha $] \mathrm{K}[\mathrm{t}]$ $\mathrm{L}[\mathrm{t}]) /(1+\backslash[$ Alpha] H K[t] $)-\backslash[$ Delta] K[t], D $[\mathrm{L}[\mathrm{t}], \mathrm{t}]==\mathrm{r} \mathrm{L}[\mathrm{t}](1-\mathrm{L}[\mathrm{t}] / \backslash[$ Eta $] 2)+(\backslash[$ Gamma $]$ $\backslash[$ Alpha] K[t] L[t] $) /(1+\backslash[$ Alpha $] \mathrm{H} \mathrm{K}[\mathrm{t}])-\backslash[$ Sigma $] \mathrm{L}[\mathrm{t}], \mathrm{K}[0]==1, \mathrm{~L}[0]==1\},\{\mathrm{K}[\mathrm{t}], \mathrm{L}[\mathrm{t}]\},\{\mathrm{t}, 0$, $300\},\{\mathrm{H}, \backslash[$ Alpha $]\}$, MaxSteps $\rightarrow \backslash[$ Infinity $]]$

ParametricPlot3D[Evaluate $\left[\left\{(\mathrm{K}[\mathrm{t}][1,8])^{\wedge} 0.4(\mathrm{~L}[\mathrm{t}][1,8])^{\wedge} 0.6,(\mathrm{~K}[\mathrm{t}][1,8]),(\mathrm{L}[\mathrm{t}][1,8])\right\} /\right.$. solH1], $\{\mathrm{t}$, 0, 100\}, PlotTheme $\rightarrow$ "Marketing", PlotRange $\rightarrow$ All, AxesLabel $\rightarrow\{$ "P[K,L]", "K[t]", "L[t]"\}] ParametricPlot3D[Evaluate $\left[\left\{0.584(1.0183)^{\wedge} \mathrm{t}\left(0.519(\mathrm{~K}[\mathrm{t}][1,8])^{\wedge}-0.756+0.481(\mathrm{~L}[\mathrm{t}][1,8])^{\wedge}-\right.\right.\right.$ 0.756)^-1.822, $(\mathrm{K}[\mathrm{t}][1,8]),(\mathrm{L}[\mathrm{t}][1,8])\} \quad$. solH1], $\{\mathrm{t}, \quad 0,100\}$, PlotTheme $\rightarrow " M a r k e t i n g "$, PlotRange $\rightarrow$ All, AxesLabel $\rightarrow\{" P[K, L] ", ~ " K[t] ", ~ " L[t] "\}]$

ParametricPlot3D[Evaluate $\left[\left\{\left(0.519(\mathrm{~K}[\mathrm{t}][1,8])^{\wedge}-0.756+0.481(\mathrm{~L}[\mathrm{t}][1,8])^{\wedge}-0.756\right)^{\wedge}-1.822,(\mathrm{~K}[\mathrm{t}][1\right.\right.$, $8]),(\mathrm{L}[\mathrm{t}][1,8])\} \quad / . \quad$ solH1], $\quad\{\mathrm{t}, \quad 0, \quad 100\}, \quad$ PlotTheme $\rightarrow$ "Marketing", PlotRange $\rightarrow$ All, AxesLabel $\rightarrow\{$ "P[K,L]", "K[t]", "L[t]"\}]

\section{References}

[1] D. Cai, H.Ye and L. Gu, A generalized Solow-Swan model, Abstr. Appl. Anal. 2014 (2014), Art. ID 395089.

[2] M. Zhou, D. Cai and H. Chen, A Solow-Swan model with technological overflow and catch-up, Wuhan Univ. J. Nat. Sci. 12(6) (2007), 975-978.

[3] R.M. Solow, A contribution to the theory of economic growth, Quart. J. Econ. 70 (1956), 65-94.

[4] E. Pelinescu, The impact of human capital on economic growth, Proc. Econ. Finance. 22 (2015), 184190.

[5] L. Gallaway and V. Shukla, The neoclassical production function, Amer. Econ. Rev. 64(3) (1974), 348358.

[6] A. Hochstein, The Harrod-Domar model in a Keynesian framework, Int. Adv. Econ. Res. 23 (2017), 349-350.

[7] A.N. Link and M.V. Hasselt, A public sector knowledge production function, Econ. Lett. 175 (2019), 64-66.

[8] M.D.P.P. Romero, A.S. Braza and A. Exposito, Industry level production functions and energy use in 12 EU countries, J. Clean. Prod. 212(1) (2019), 880-892.

[9] M.D.P.P. Romero and A.S. Braza, Productive energy use and economic growth: energy, physical and human capital relationships, Energy Econ. 49 (2015), 420-429.

[10] A.J. Lotka, Elements of physical biology, first ed., Williams and Wilkins, Baltimore, 1925. 
[11]V. Volterra, Variazioni efluttuazioni del numero di individui in specie animali conviventi, Mem. Reale Accad. Naz. Lince. 2 (1926), 31-113.

[12] L. Nie, Z. Teng, L. Hu and J. Peng, The dynamics of a Lotka-Volterra predator-prey model with state dependent impulsive harvest for predator, Biosyst. 98 (2009), 67-72.

[13] B. Chakraborty and N. Bairagi, Complexity in a prey-predator model with prey refuge and diffusion, Ecol. Complexity. 37 (2019), 11-23.

[14] B. Sahoo and S. Poria, Dynamics of predator-prey system with fading memory, Appl. Math. Comput. 347(15) (2019), 319-333.

[15] A. Bouskila, Games played by predators and prey, Encyclopaedia Ani. Behav. (2019), 382-388.

[16]S.L. Tilahun, Prey predator hyperheuristic, Appl. Soft Comput. 59 (2017), 104-114.

[17] V. Castellanos and R.E.C. Lopez, Existence of limit cycles in a three level trophic chain with LotkaVolterra and Holling type II functional responses, Chaos Solitons Fractals. 95 (2017), 157-167.

[18] M. Liu, C. Du and M. Deng, Persistence and extinction of a modified Leslie-Gower Holling-type II stochastic predator-prey model with impulsive toxicant input in polluted environments, Nonlinear Anal. Hybrid Syst. 27 (2018), 177-190.

[19]Z. Xiao, X. Xie and Y. Xue, Stability and bifurcation in a Holling-type II predator-prey model with Allee effect and time delay, Adv. Difference Equ. 2018 (2018), Art. ID 288.

[20]J. Carkovs, J. Goldsteine and K. Sadurskis, The Holling-type II population model subjected to rapid random attacks of predator, J. Appl. Math. 2018 (2018), Art. ID 6146027.

[21]G.E. Vilcu, A geometric perspective on the generalized Cobb-Douglas production functions, Appl. Math. Lett. 24 (2011), 777-783.

[22] X. Wang and Y. Fu, Some characterizations of the Cobb-Douglas and CES production functions in microeconomics, Abstr. Appl. Anal. (2013), Art. ID 761832.

[23]K.J. Arrow, H.B. Chenery, B.S. Minhas and R.M. Solow, Capital-labour substitution and economic efficiency, Rev. Econ. Statist. 43(3) (1961), 225-250.

[24]K. Sato, A two-level constant-elasticity of substitution production function, Rev. Econ. Stud. 34(2) (1967), 201-218. 


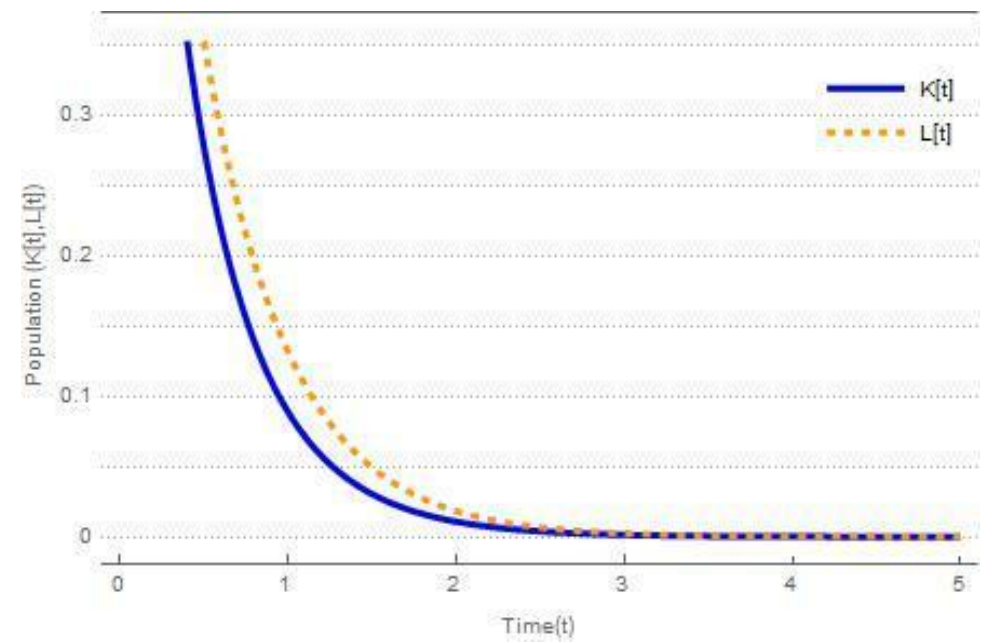

Figure 2. Solutions of Eq. (1) showing trivial equilibrium point when $s=2, r=2, \eta_{1}=3$,

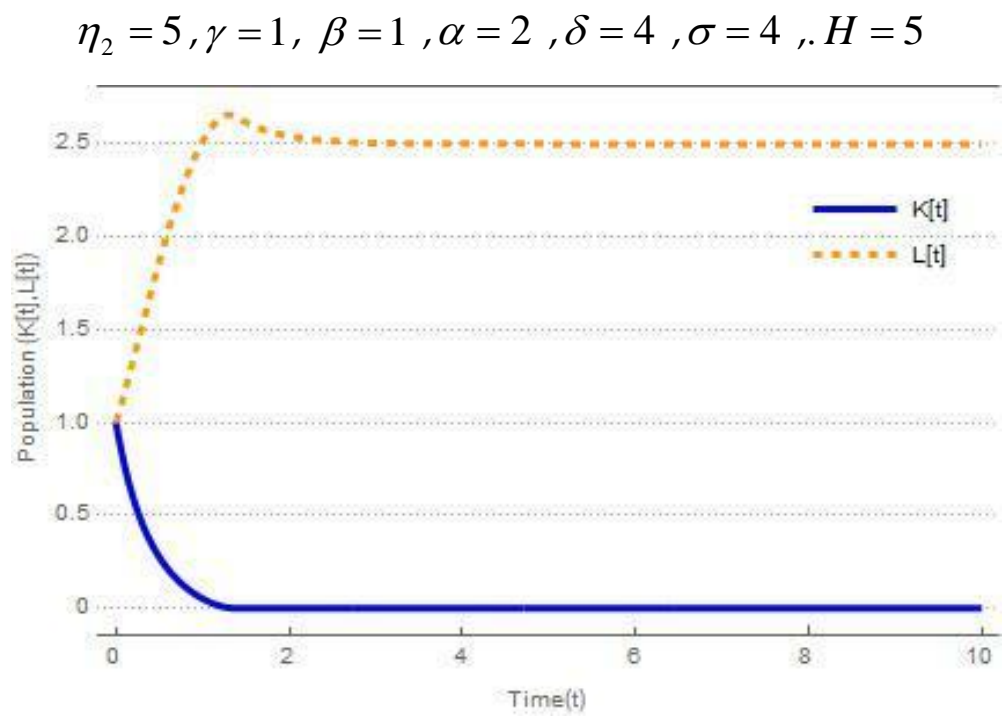

Figure 3. Solutions of Eq. (1) showing prey free equilibrium point when $s=2, r=4, \eta_{1}=3$,

$$
\eta_{2}=5, \gamma=6, \beta=1, \alpha=8, \delta=4, \sigma=2, . H=15
$$




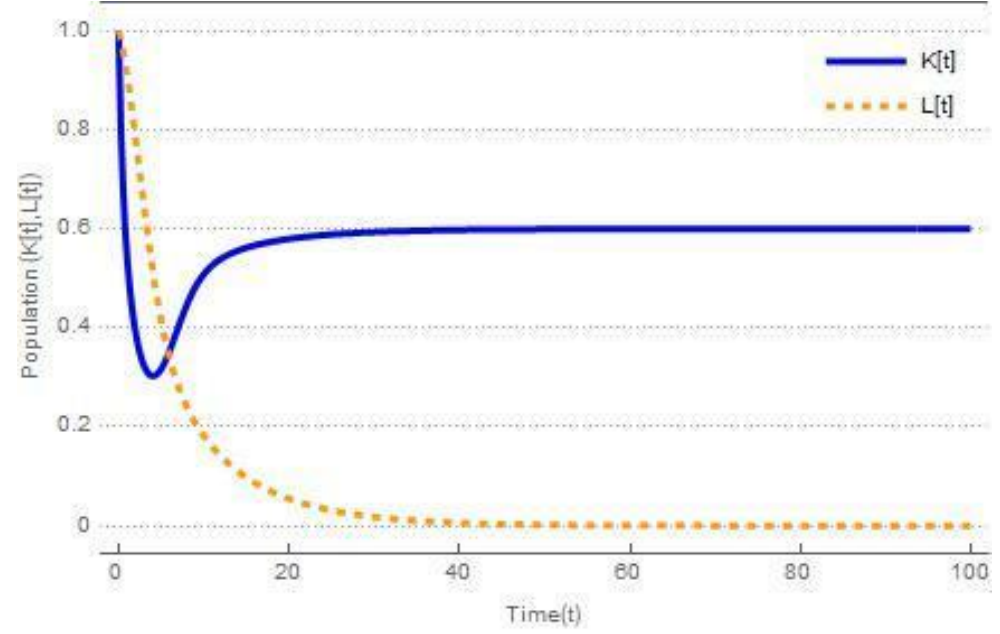

Figure 4. Solutions of Eq. (1) showing predator-free equilibrium point when $s=5, r=002$,

$$
\eta_{1}=3, \eta_{2}=5, \gamma=6, \beta=1, \alpha=8, \delta=4, \sigma=2, . H=3
$$

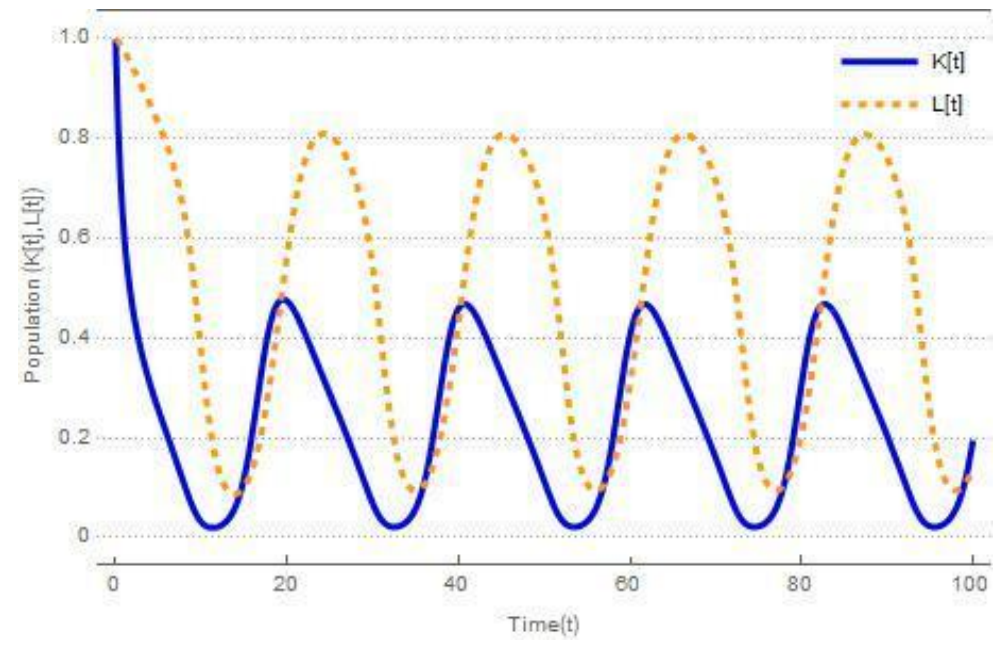

Figure 5. Solutions of Eq. (1) showing unstable fluctuations of coexistence equilibrium point when $s=3, r=2, \eta_{1}=1, \eta_{2}=3, \gamma=6, \beta=1, \alpha=8, \delta=2, \sigma=3, . H=3.5$ 


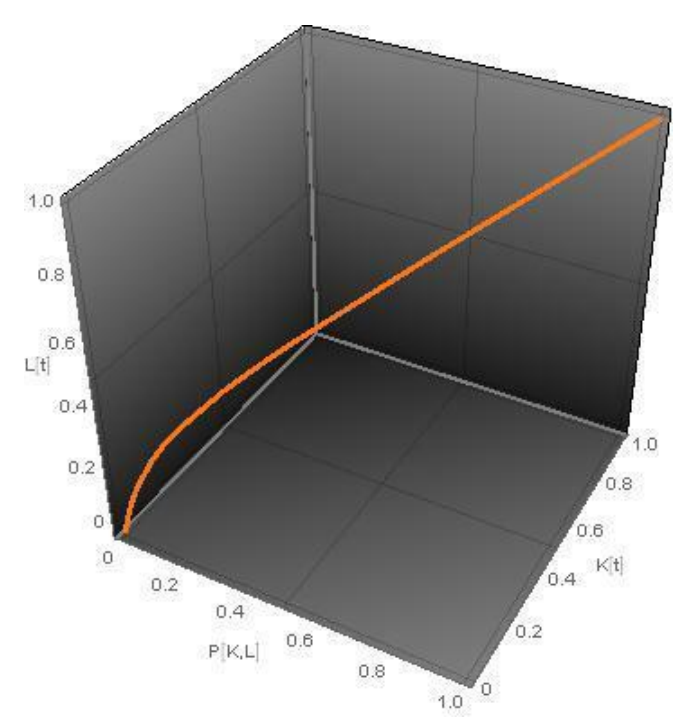

Figure 6. Cobb-Douglas production function [22] for $t \in[0,100], \eta_{1}=2, \eta_{2}=3, \gamma=6, \beta=1$, $\alpha=8, H=3.5$ when $. \delta=\sigma=4, s=r=2$

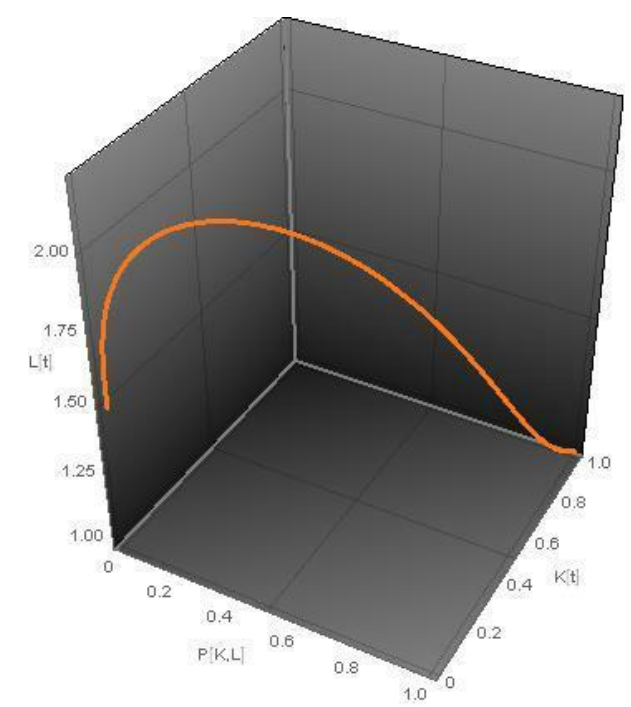

Figure 7. Cobb-Douglas production function [22] for $t \in[0,100], \eta_{1}=2, \eta_{2}=3, \gamma=6, \beta=1$, $\alpha=8, H=3.5$ when $. \delta=4, \sigma=2, s=2, r=4$ 


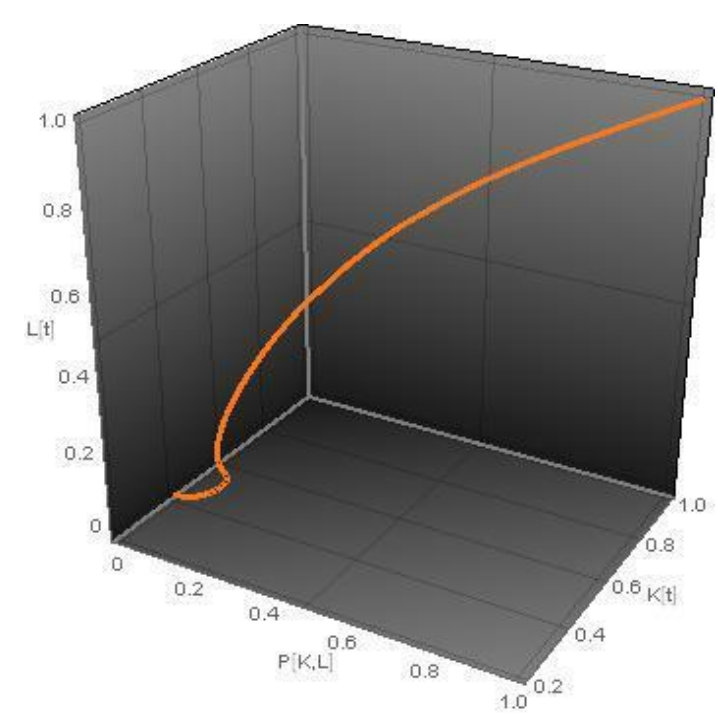

Figure 8. Cobb-Douglas production function [22] for $t \in[0,100], \eta_{1}=2, \eta_{2}=3, \gamma=6, \beta=1$, $\alpha=8, H=3.5$ when $\delta=4, \sigma=2, s=5, r=0.316$

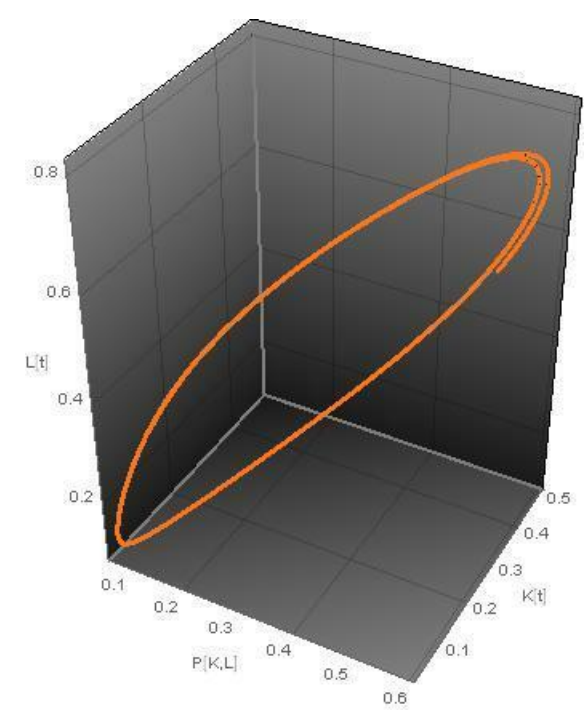

Figure 9. Cobb-Douglas production function [22] for $t \in[0,100], \eta_{1}=2, \eta_{2}=3, \gamma=6, \beta=1$, $\alpha=8, H=3.5$ when $. \delta=2, \sigma=3, s=3, r=2$ 


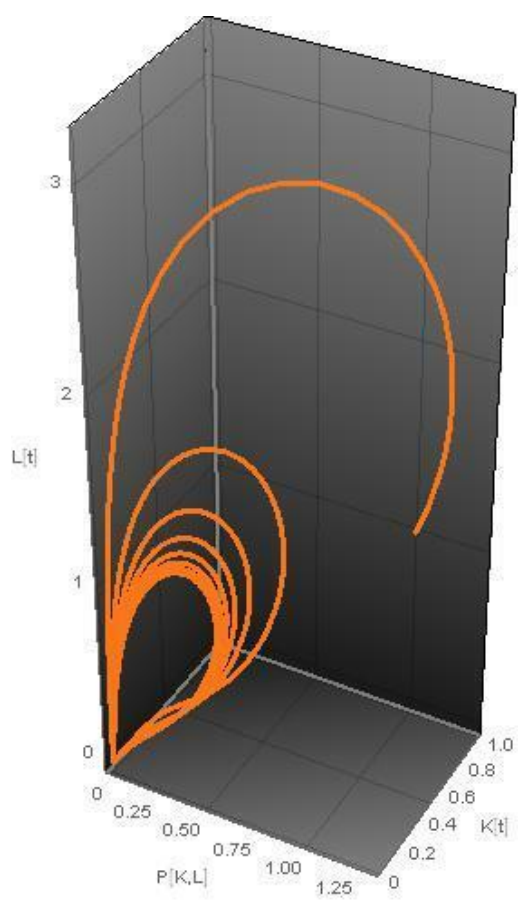

(a) Production function [22] with $\Omega=1$ and $\rho=0.4$.

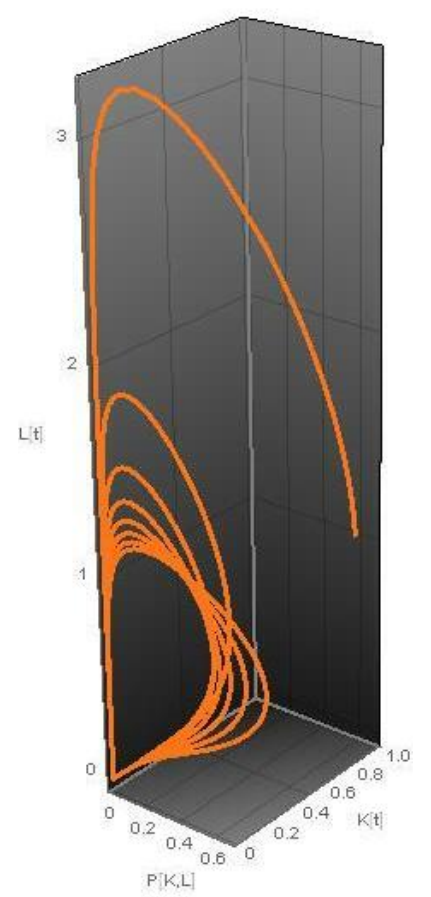

(b) Production function [23] with $\Pi=0.584, \omega=0.519$ and $\rho=0.756$. 


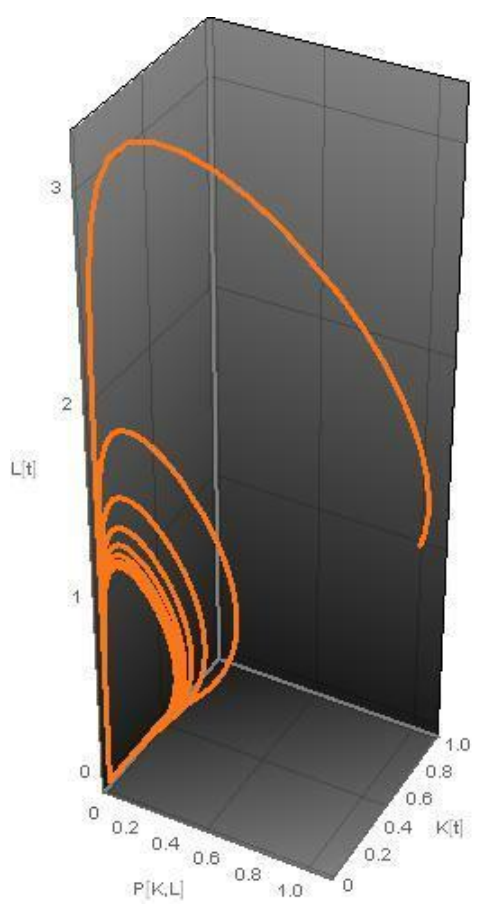

(c) Production function [24] with $\Pi=1, \omega=0.519$ and $\rho=0.756$.

Figure 10(a-c). Different production functions [22-24] for, $\eta_{1}=2, \eta_{2}=3, \gamma=6, \beta=1, \delta=2$,

$$
\sigma=3, r=2, . H=1 \text { and } \alpha=8 \text { when } s=3
$$

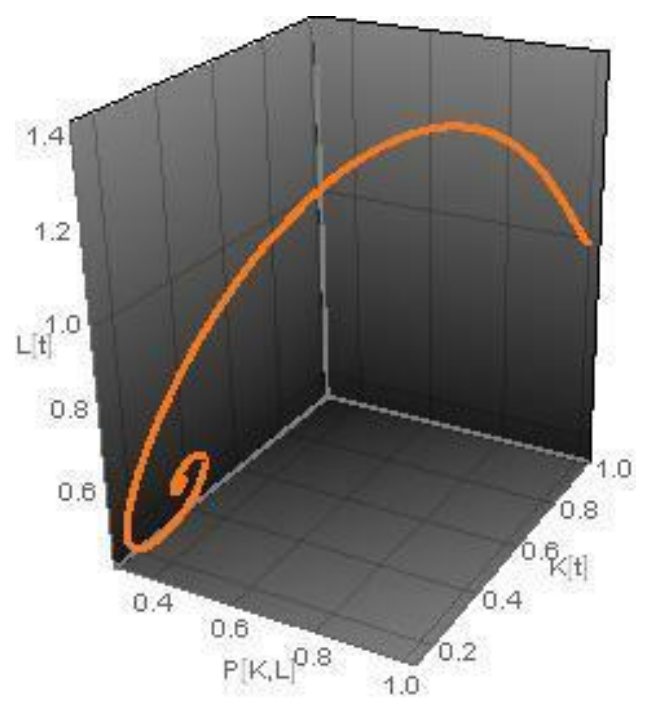

(a) Production function [22] with $\Omega=1$ and $\rho=0.4$. 


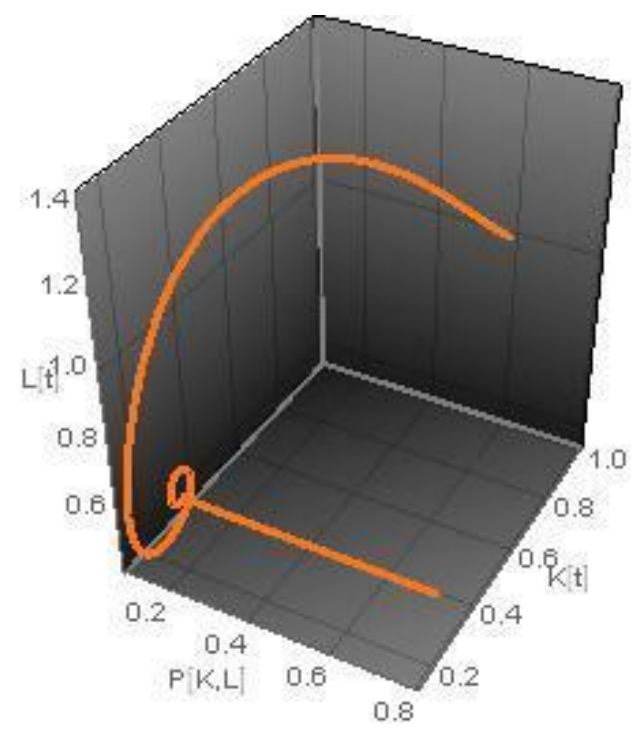

(b) Production function [23] with $\Pi=0.584, \omega=0.519$ and $\rho=0.756$.

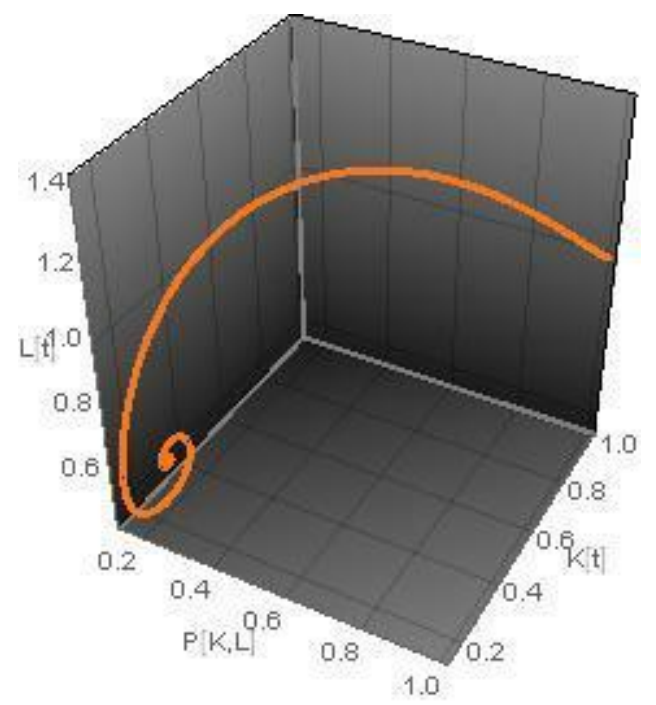

(c) Production function [24] with $\Pi=1, \omega=0.519$ and $\rho=0.756$.

Figure 11(a-c). Different production functions [22-24] for , $\eta_{1}=2, \eta_{2}=3, \gamma=6, \beta=1, \delta=2$,

$$
\sigma=3, r=2, . H=2 \text { and } \alpha=2 \text { when } s=3
$$




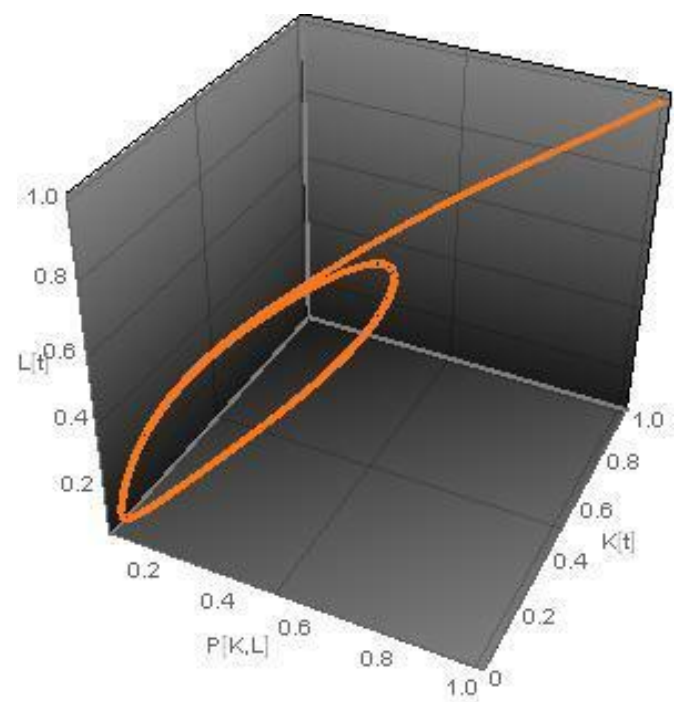

(a) Production function [22] with $\Omega=1$ and $\rho=0.4$.

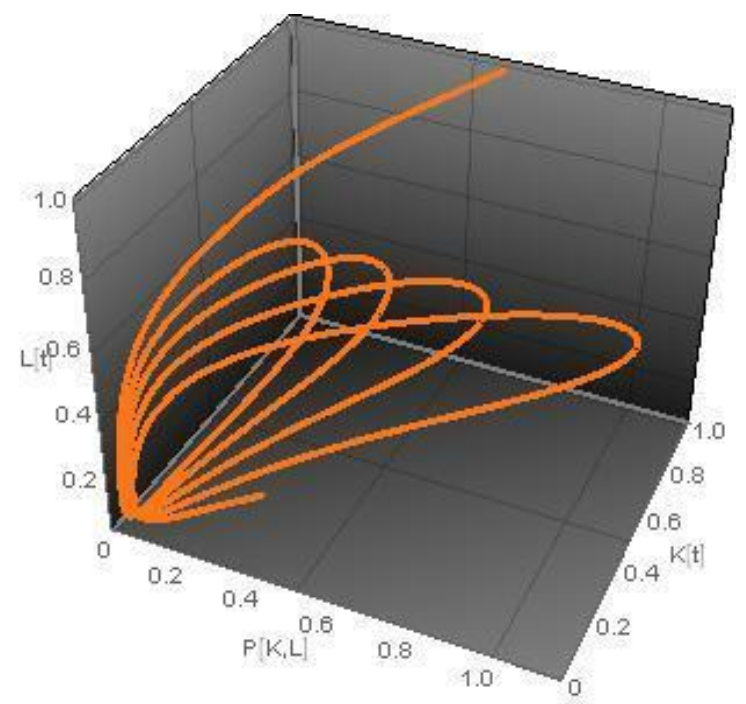

(b) Production function [23] with $\Pi=0.584, \omega=0.519$ and $\rho=0.756$. 


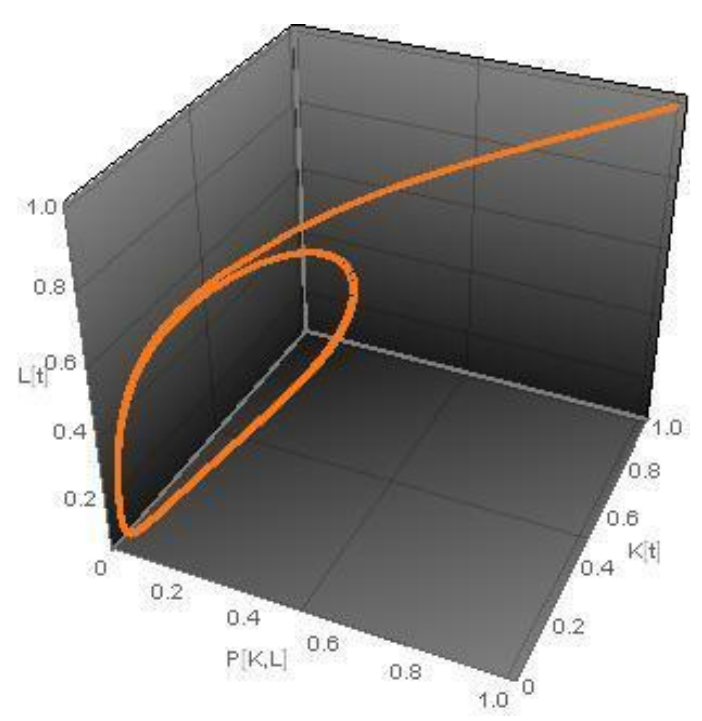

(c) Production function [24] with $\Pi=1, \omega=0.519$ and $\rho=0.756$.

Figure 12(a-c). Different production functions [22-24] for, $\eta_{1}=2, \eta_{2}=3, \gamma=6, \beta=1, \delta=2$,

$$
\sigma=3, r=2, H=3.5 \text { and } \alpha=8 \text { when } s=3
$$

